\title{
Preliminary findings on competition in healthcare should not be over-interpreted
}

\author{
Nick Black professor of health services research \\ London School of Hygiene and Tropical Medicine, London WC1E 7HT, UK
}

Research into the effects of competition on quality of care must guard against the tendency to make over-optimistic claims of benefits on the basis of initial findings. ${ }^{1}$

Despite the best attempts of excellent researchers, the three existing studies in England are inevitably limited by their reliance on routine administrative data (hospital episode statistics; HES) and the need to make some heroic but dubious assumptions (such as acute myocardial infarctions are "easily clinically identifiable," "adjusted mortality rates are purged of case-mix"). ${ }^{2-4}$ These studies also mostly focus on one aspect of quality - safety (hospital mortality rates and case fatality rates) — and pay little attention to effectiveness, humanity (experience), or equity.

The findings of these studies need to be interpreted more cautiously. For example, the strongest evidence concerns acute myocardial infarction case fatality rates. ${ }^{3}$ The key finding, a dramatic decrease from 2002 to 2008, occurred in all hospitals during a time when central policies such as the national service framework and National Institute for Health and Clinical Excellence guidelines were being implemented and additional resources provided. If all methodological concerns are ignored, the research shows that for a short period (2007) hospitals in more competitive areas improved faster, although this was not apparent in 2008. This suggests that, for one specific condition, competition produced a marginal benefit, but it does not warrant the claim of "death by market power."

If competition can help improve NHS quality, then research must be conducted in other clinical areas using data that are not subject to the limitations of HES. Until we have a clearer picture, researchers should not encourage policy makers to over-interpret interesting but preliminary findings.

Competing interests: None declared.

Mays N. Is there evidence that competition in healthcare is a good thing? No. BMJ 2011;343:d4205. (5 July.)

2 Gaynor M, Moreno-Serra R, Propper C. Death by market power: reform, competition and patient outcomes in the National Health Service. July 2010. University of Bristol CMPO Working Paper 10/242.

3 Cooper Z, Gibbons S, Jones S, McGuire A. Does hospital competition save lives? Evidence from the English NHA patient choice reforms. Econom $J$ [forthcoming].

4 Seiler S. Free to choose? Reform and demand response in the British National Health Service [PhD thesis]. London School of Economics, 2011. 